\title{
Jean-Louis Prévost (1790-1850) et la découverte de l'ovule des mammifères
}

\author{
Par G. DE Morsier, Genève
}

Prévost et Dumas ont abordé l'étude, pleine de difficultés, de la fécondation chez les mammifères, lapine et chienne, dans leur $3^{\mathrm{e}}$ mémoire de1824: Vingt-quatre heures après la copulation, les spermatozoïdes sont très nombreux dans les cornes de l'utérus, mais absents dans les ovaires. On voit des ovules libres dans les cornes, très petits $(1 \mathrm{~mm})$, comme de GraAF et Cruikshank l'avaient déjà signalé. Le moment de la fécondation est de beaucoup postérieur à celui de la copulation. L'ovule est mis en liberté par l'ouverture des capsules (follicules) de l'ovaire, il est reçu par le pavillon des trompes et amené dans les cornes de l'utérus où il est fécondé par le spermatozoïde. Prévost et Dumas ont vu aussi l'ovule de la chienne non fécondé. «Il nous est survenu deux fois, en ouvrant des vésicules (follicules) très avancées de rencontrer dans leur intérieur un petit corps sphérique d'un millimètre de diamètre. Mais il différait des ovules que nous observeons dans les cornes par sa transparence qui était beaucoup moindre.» A cause de cette différence d'opacité, les auteurs estiment que de nouvelles recherches sont nécessaires avant de pouvoir affirmer qu'il s'agit bien de l'ovule. La planche $\mathrm{V}$ montre le développement de l'œuf à partir du $8^{\mathrm{e}}$ jour après la copulation. Dans cet exposé, tout est clair sauf le diamètre de l'ovule qui, en réalité, est environ cinq fois plus petit qu'un millimètre.

Après que Dumas ait quitté Genève en 1823, Prévost reprend seul l'étude de la génération chez les mammifères, comme il l'avait annoncé. Voici ce qu'il a observé chez la vache: "Au moment où la vésicule de l'ovaire se rompt, il s'écoule un liquide qui entraîne avec lui dans la trompe de Fallope et de là dans l'utérus un globule (ovule), mais entièrement dégagé de tout appendice nutritif, nous avons déjà parlé de ce globule dans notre Mémoire avec M. Dumas, inséré au $3^{\mathrm{e}}$ volume des Annales des Sciences Naturelles, page 113. J'ai désiré l'étudier sur les ovaires des vaches; en conséquence, j'en ai pris un certain nombre, j'ai ouvert les vésicules qu'ils portaient, recueilli le liquide contenu sur un porte-objet: l'on y voyait flotter de petits débris membraneux, que j'ai examinés un à un au microscope; dans plusieurs cas, cette investigation minutieuse m'a réussi; $j$ 'ai retrouvé un globule bien dessiné, analogue à ceux que j’avais auparavant observés: il était fixé 
dans une portion de membrane plus ou moins considérable: il s'est toujours trouvé unique pour chaque vésicule de l'ovaire; quant à sa grosseur, elle variait suivant les cas entre $0,16 \mathrm{~mm}$ et $0,30 \mathrm{~mm}$ de diamètre; il était régulièrement sphérique; il offrait à sa surface une portion circulaire plus transparente; c'est le lieu où plus tard se montrent les premiers rudiments du foetus. Le globule passe dans l'utérus, la fécondation s'opère; le foetus paraît».

Prévost a donc confirmé sur la vache ce qu'il avait déjà observé chez la lapine et chez la chienne, en précisant le diamètre de l'ovule non fécondé, qui est maintenant exact, et en décrivant le noyau. On doit donc se demander si J.L.Prévost a découvert l'ovule des mammifères avant KARL voN BAEr. Le travail que nous venons de citer a paru dans les Mémoires de la Société de Physique et d'Histoire Naturelle de Genève, tome IV, partie I, page 60 , qui porte la date de 1828 , mais il a certainement été écrit avant, car la partie I ne contient que des mémoires lus à la Société de 1824 à 1826. Ce retard s'explique facilement, car les Mémoires de la Société ne paraissent que tous les quatre ans: 1821, 1824, 1828, 1832. Ainsi le mémoire de Prévost sur la fécondation chez les gastéropodes, lu en 1828, n'a paru qu'en 1832. D'autre part, une note du mémoire de 1825 sur le poisson «Séchot» doit faire admettre qu'à cette date Prévost avait déjà étudié la fécondation chez la vache ${ }^{1}$.

J'ai recherché dans les Comptes rendus (manuscrits) de la Société de Physique de Genève les dates exactes de la lecture de ces notes. Voici ce que j'ai trouvé:

«Séance du 19 avril 1827. - M. le D Prévost dit avoir observé dans le fluide qui remplit les vésicules (follicules) de l'ovaire d'une vache des agglomérations de globules offrant de temps à autre une cicatricule unique dans chaque vésicule et de grosseur variable; il montre des dessins de ces cicatricules.»

Les dessins n'ont pas été retrouvés. Les termes «globule» et «cicatricule» sont synonymes de «ovule»; Prévost les emploie souvent dans ce sens. Etant donné la terminologie imprécise de l'époque, le botaniste JaCquesDenis Choisy, alors secrétaire de la Société, ne pouvait pas être beaucoup plus précis.

1 «Je dois à l'obligeance de M.Morin, chimiste très distingué de notre ville, les observations suivantes sur les propriétés chimiques des contenus de l'œuf des séchots. Ils présentent infiniment d'analogie avec les jaunes d'œufs de poule, et les corps jaunes de l'ovaire de la vache. " (De la génération chez le séchot, Mémoires de la Société de Physique et d'Histoire Naturelle de Genève, tome IV, 1828, note de la page 174. (ce mémoire, paru seulement en 1828, a été lu en 1825.) 
«Séance du 16 août 1827. - M. le $\mathrm{D}^{r}$ Prévost lit un Note sur la circulation du fœtus dans l'utérus. L'observation a été faite sur une brebis; elle confirme l'opinion déjà émise par l'auteur, que le fœtus a une circulation qui lui est propre, chez les vivipares comme chez les ovipares. Pour le reconnaître, il faut observer de bonne heure, avant qu'il y ait une adhérence entre les membranes des deux êtres.» Il est évident qu'en 1828 Prévost a publié sous un même titre (parce qu'il s'agissait de Ruminants) les deux notes lues en 1827.

Comme Karl von Baer l'a raconté lui-même en 1866, il a vu l'ovulenon fécondé de la chienne au printemps 1827. Il l'a décrit et figuré dans sa lettre à l'Académie de St-Petersbourg (De ovi mammalium et hominis genesi epistola), qui est datée de Prid.Iduum Quinct, c'est-à-dire de la veille du 15 juillet 1827. Baer cite à plusieurs reprises le mémoire de Prévost et Dumas de 1824, dont il reproduit dans sa planche deux figures (6 et VI). Dans la seconde partie de son livre Über Entwicklungsgeschichte der Thiere, écrite en 1834 et parue en 1837, il dit dans une note au pas de la page 173: «Prévost et Dumas paraissent avoir découvert l'ovule de la chienne, mais sans le considérer comme tel», puis il cite le mémoire de Prévost sur l'ovule de la vache en disant que sa description est exacte, mais il se trompe en datant ce mémoire de 1829: c'est en 1828 qu'il a paru dans les Mémoires de la Société de Physique de Genève (celui des Annales des Sciences naturelles paru en 1829 n'est qu'une reproduction). Il ne pouvait pas savoir que la découverte avait été annoncée le 19 avril 1827.

On doit donc conclure que la découverte de l'ovule des mammifères, attribuée généralement à K. von Baer, appartient en réalité à Prévost.

Dans un remarquable travail, Heinrich Buess est déjà arrivé aux mêmes conclusions, sans citer le travail de Prévost sur l'ovule de la vache (1947). Ce que nous venons d'exposer confirme donc complètement son opinion. A propos de l'iconographie des travaux de Prévost, dont Buess regrette l'insuffisance, je signale l'existence d'un Atlas imprimé in $4^{\circ}$, contenant 22 planches, annexé aux Annales des Sciences naturelles, pour les tomes 1, 2, 3 et 12, dans lequel les tirages des lithographies sont meilleurs que dans les volumes correspondants des Annales et qui sont accompagnés delégen des beaucoup plus explicites ${ }^{2}$. Avec raison, Buess relève la trop grande mo-

2 Un exemplaire de cet Atlas se trouve au Musée de l'Histoire des Sciences de Genève. J'ignore s'il s'agit d'une épreuve, d'un exemplaire isolé ou s'il a été annexé régulièrement aux tomes correspondants des Annales. 
destie des deux biologistes de Genève, qui n'ont pas mis en évidence l'importance immense de leur découverte. Cependant, il semble bien qu'ils l'aient comprise. En février 1825, Dumas écrit dans l'article «Génération » du Dictionnaire classique d'histoire naturelle: «Ainsi se trouve expliquée l'influence particulière du mâle et de la femelle dans la procréation de l'être auquel ils donnent naissance, ainsi se trouvent expliquées toutes les ressemblances héréditaires qui ont tant occupé les physiologistes du siècle dernier.» Il faut aussi rappeler que Prévost n'a pas occupé de chaire universitaire, n'a pas fait d'enseignement et n'a pas eu d'élèves. Très modeste, il n'a pas publié de Traité d'Embryologie pour exposer l'ensemble de ses travaux, comme l'ont fait K. von Baer et plus tard O. Hertwig, et il n'a jamais revendiqué la priorité de ses découvertes. En matière de découverte scientifique, la publicité a malheureusement beaucoup d'importance et c'est l'historien des Sciences qui doit rétablir les faits ${ }^{3}$. Prévost et Dumas avaient une supériorité certaine sur l'école de Pander et v. Baer: ils étaient à la fois morphologistes, physiologistes et chimistes. Baer croyait encore que les animalcules spermatiques sont des infusoires parasites.

Prévost et Dumas, puis Dumas seul, ont fait les découvertes suivantes concernant la génération:

1. Les spermatozoïdes proviennent uniquement des testicules.

2. L'ovule des mammifères, dont le diamètre est de 0,16 à $0,30 \mathrm{~mm}$, se trouve dans le follicule de Graaf, à raison d'un seul ovule par follicule. Après rupture de ce dernier, l'ovule parvient dans la trompe et la corne de l'utérus, endroits où s'opère la fécondation. Elle ne se produit jamais dans l'ovaire.

3 D'après Buess, W. von Buddenbrock prétend dans Bilder aus der Geschichte der Biologischen Grundlagen (1930) que la segmentation de l'œuf a été découverte par v.BAER, ce qui prouve qu'il n'a pas lu Prévost et Dumas. J'ai constaté qu'Oscar Hertwig, dont le Précis d'Embryologie (1886) a eu un grand nombre d'éditions et a été traduit dans plusieurs langues, ne mentionne pas PrÉvost et affirme que SPALLANZANI a découvert que le contact entre le spermatozoïde et l'ovule est nécessaire pour la fécondation! Charbes SINGER, dont le livre History of Biology est très répandu et a été traduit, ne mentionne pas Prévost et Dumas, alors qu'il consacre 6 pages à v. Baer. Même erreur dans J. Needham (History of Embryology, 1959). A Genève même, Hermann Fol répète l'erreur d'Hertwig (Recherches sur la fécondation, 1879, p. 213). Depuis K.von BaEr (1837) aucun auteur, à ma connaissance, n'a cité le travail fondamental de PrÉvost sur l'ovule de la vache. 
3. La fécondation n'est possible que si le spermatozoïde est en contact intime avec l'ovule. Le sperme dépourvu de spermatozoïde n'a aucune action fécondante.

4. L'œuf fécondé de la grenouille se segmente en deux, quatre, huit, seize parties et ainsi de suite, puis prend l'aspect d'une framboise (morule). L’embryon se développe ensuite autour de la cicatricule.

5. Les jeunes embryons des différentes classes des vertébrés ont un aspect identique (loi dite «biogénétique»).

6. En modifiant les conditions physiques et chimiques des œufs de poules (température, atmosphère, état électrique), on peut produire à volonté des monstres.

En 1877, le Genevois Hermann Fol verra le premier la pénétration du spermatozoïde dans l'ovule chez Asterias glacialis.

Ces découvertes sont à la base de la Biologie et de la Génétique moderne.

\section{Bibliographie}

\section{Manuscrits}

Prévost Dr., Ciompte rendu des séances du 19 Avril et du 16 Août 1827 de la Société de Physique et d'Histoire Naturelle de Genève, Bibliothèque de Genève.

\section{Imprimés}

Baer Karl Ernst von, De ovi mammalium et hominis genesi... Lipsiae, Sumptibus L. Vossii, 1827.

- Über Entwickelungsgeschichte der Thiere. Beobachtungen und Reflexion, Königsberg (Geb. Bornträger). I. Theil 1828, II. Theil 1837, p. 175.

- Nachrichten über Leben und Schriften (Mitgetheilt von ihm selbst), St. Petersburg 1864, p. 306 .

Buess Heinrich, The Contribution of Geneva Physicians to the Physiology of Development in the 19th Century, Bull.Hist. Med.31 (1947) 871-897.

Cruikshank W., Versuche bei Welchen die Eyer der Kaninschen am dritten Tage nach der Begattung in der Gebährmutter gefunden wurden; nebst der ersten Gestalt der Frucht, Phil.Trans. Roy. Soc. (London) I (1797) 197, und Arch. Physiol. (Reil) 3 (1799) 74-94.

D(umas). J. B., Génération, in Dictionnaire Classique d'Histoire Naturelle, tome 7, p. 194-222, Rey et Gravier, Paris 1825.

Prévost et Dumas, Troisième mémoire: De la génération dans les Mammifères et des premiers indices du développement de l'Embryon, Ann. Sci. Nat. 3, tome III (1824) 113-138.

- Nouvelle théorie de la génération. Physiologie et anatomie animale, Atlas accompagnant les Annales des Sciences Naturelles 1, 2, 3 et 12 (1824-1827), 22 planches, s.d.

Prévost Docteur. Note sur la Circulation du Foetus chez les Ruminants, Mémoires de la Société de Physique et d'Histoire naturelle de Genève IV (1828), première partie, p. 60-66 (note reproduite dans les Ann.Sci.Nat. 16 [1829] 156-162). 


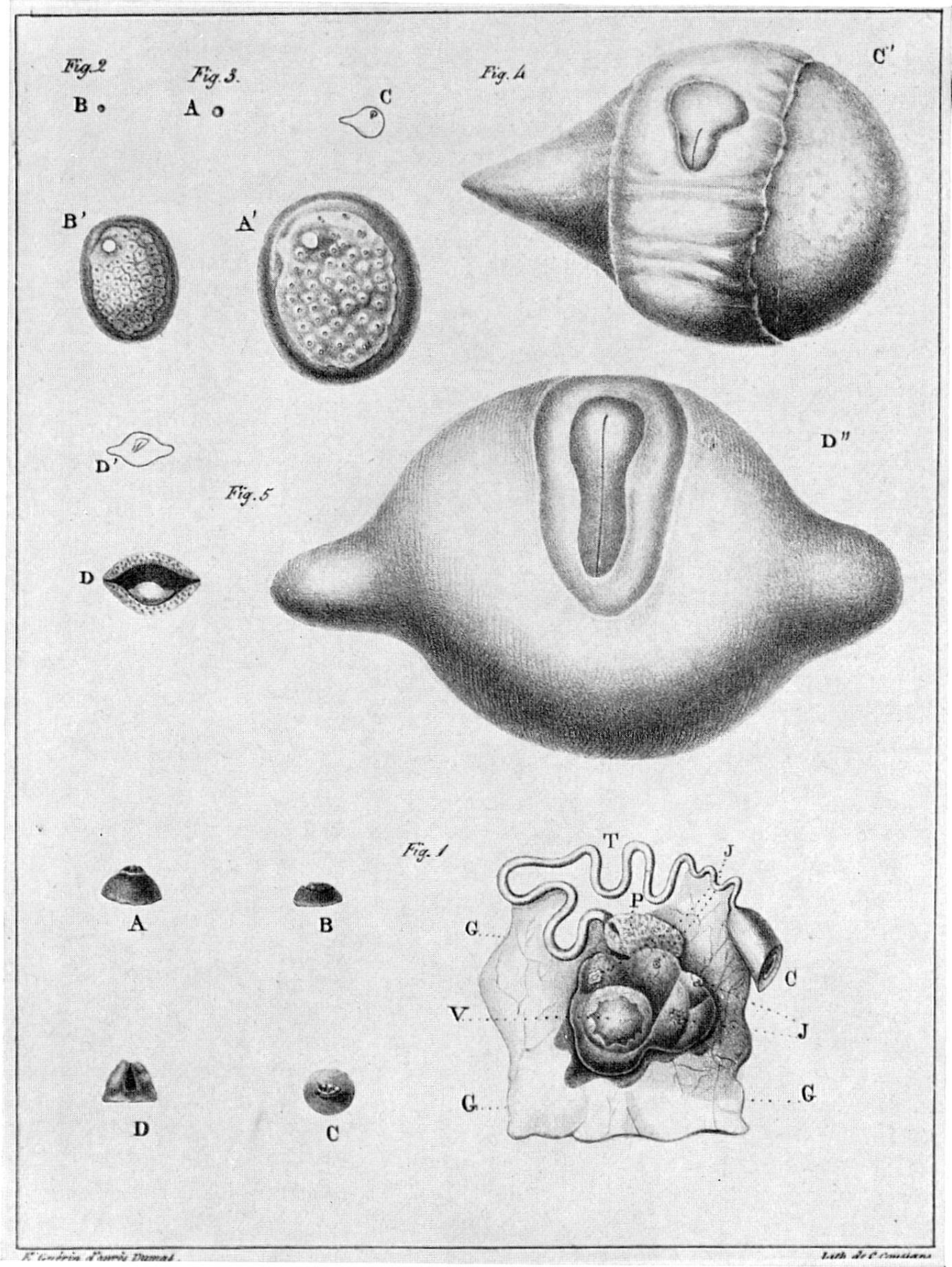

Légende voir page suivante 
A gauche: Planche V (d'après Dumas): Corps jaune et ovule

Fig. 1. Ovaire huit jours après l'accouplement; $\mathrm{C}$ cornes; $\mathrm{T}$ trompe; $\mathrm{P}$ pavillon; GGG graisse; $\mathrm{V}$ grosse vésicule près de s'ouvrir; $\mathrm{J}$ corps jaune; $\mathrm{A} B$ corps jaune vu de côté; $\mathrm{C}$ idem vu par dessus; $D$ idem ouvert pour voir le fond de la cavité

Fig. 2. Ovule de la même époque, vu de grandeur naturelle en B, et grossi en B'

Fig. 3. Ovule pris sur le même animal, mais plus près de la base des cornes. De grandeur naturelle en $\mathbf{A}$, grossi en $\mathbf{A}^{\prime}$

Fig. 4. C ovule de douze jours, de grandeur naturelle, $\mathrm{C}^{\prime}$ le même grossi

Fig. 5 . D ovule de même date, plus avancé que le précédent et en place; $D^{\prime}$ séparé de la corne, $\mathrm{D}^{\prime \prime}$ grossi 